Decano Facultad de Ciencias Médicas. UNC

Prof. Dr. Rogelio D. Pizzi

Vice Decana Facultad de Cs. Médicas. UNC

Prof. Dra. Marta Fiol de Cuneo.

Secretaría de Salud Pública y Ambiente

Prof. Dr Gustavo Irico

Directora de la Revista

Prof. Dra. Ma. Cristina Cometto

\section{Comité editorial}

Prof. Mg. María Borsotti

Escuela de Salud Pública. FCM UNC

Prof. Dra. Patricia F. Gómez

Escuela de Enfermería FCM UNC.

Prof. Med. Gustavo Martínez

Municipalidad de Córdoba

Prof. Mg. Rubén Castro Toschi

Esc. de Salud Pública. FCM UNC

\section{Comité de Redacción}

Prof. Dr. Leandro Dionisio

Esc. de Salud Pública. FCM UNC

Med. Mariela Roldan

Hospital Nacional de Clínicas

Mg. Susana Rivolta

Esc. de Salud Pública FCM UNC

Dr. Ariel Abeldaño

Universidad de la Sierra Sur - Oaxaca, México

Dra. Monica Bella

Esc. de Salud Pública FCM UNC

Mg. Hugo L. Pizzi

Esc. de Salud Pública FCM UNC

\section{Consejo Científico Nacional}

Prof. Dra. Noemí Bordoni. Directora del Instituto de Investigaciones en Salud Pública. Coordinadora Ejecutiva de la Maestría en Salud Pública. UBA

Prof. Dra. Norma Cristina Meichtry. Doctor of Philosophy (Sociología- Demografía). Profesora Titular Ordinaria en Geografía Argentina UNNE. Directora Instituto de Revista de Salud Pública, (XIX) 1:1, abr 2015 Investigaciones Geohistóricas. Investigadora Independiente en el Inst. de Investigaciones Geohistóricas. CONICET

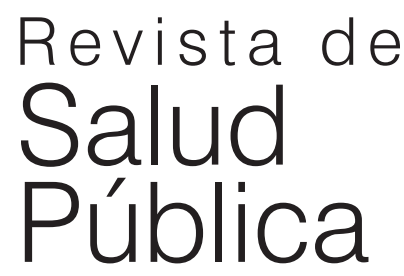

Dr. Jorge Kiguen Prof. Titular Ordinario de Epidemiologia. Universidad Nacional del Litoral. Rep. A.

Prof. Mg Ana M Heredia. Profesor Titular Regular Escuela de Enfermería. Departamento de Ciencias Sociales. Universidad Nacional de Quilmes

Dra. Silvina Malvarez. Ex consultora de la OPS OMS WDC

Dr. Luis Simes. Instituto Universitario de Ciencias de la salud. Universidad Barceló. Bs. As.

Dr. Bruno Sebastián Ribotta. Investigador Asistente del CONICET. Lugar de trabajo Centro de Investigaciones y Estudio sobre Cultura y Sociedad. CIECS, CONICET y UNC. Universidad Nacional de Córdoba.

\section{Consejo Científico Internacional}

Prof. Dr. Giorgio Solimano. Universidad de Chile

Dr. Charles Godue. Ex consultor OPS. WDC

Prof. Dr. Juan Jesús Gestal Otero. Universidad de Sgo. de Compostela. España

Prof. Dr. José Ramón Martínez Riera. Profesor Titular del Depto. de Enfermería Comunitaria. Medicina Preventiva y Salud Pública e Historia de la Ciencia de la Universidad de Alicante. España.

Prof. Rosa A. Zarate Grajales.

Escuela de Enfermería y Obstetricia. Universidad Nacional Autonoma México. 
La Revista de Salud Pública es una publicación temática, indizada, con arbitraje externo de pares nacionales e internacionales, trimestral, destinada a la publicación de la producción técnico científica relacionada al área de la salud y en especial de la Salud Pública. Editada por la Escuela de Salud Pública de la Facultad de Ciencias Médicas de la Universidad Nacional de Córdoba.

Consta de ISSN 1853-1180 para la versión Impresa y ISSN 1852-9429 para la Electrónica.

The Journal of Public Health is a thematic publication, indexed, external peer refereed national and international, biannual, for the publication of the technical-scientific production related to the area of health and in particular Public Health. Published by the School of Public Health, Faculty of Medical Sciences of the University National Córdoba, ISSN $1853-1180$ consists of the printedversion and for Electronic ISSN 1852-9429.

Bibliotecóloga: Mónica S. Perfetti

Traducción al inglés: Andrea Ternengo

Traducción al portugués: Osvaldo J. Casero

Tesorería y Finanzas: Cdora. Soledad Marquez

Diseño y Diagramación: Vladimir López Barrios

Escuela de Salud Pública. Calle Bv. de la Reforma esq. Enf. Gordillo Gomez CP 5000. Córdoba. República Argentina. Te: 0351 - 4334042 / 4333023

www.saludpublica.fem.unc.edu.ar saludpublica@fcm.unc.edu.ar publicacionesp@fcm.unc.edu.ar ISSN 1853-1180

Las responsabilidades por los juicios, opiniones, puntos de vista, o traducciones; expresados en los artículos publicados corresponden exclusivamente a los autores.

No está permitida la reproducción parcial o total del contenido de la revista, sin la previa autorización de los editores de la Revista de Salud Pública.

Impreso en:

Taller General de Imprenta, UNC Marzo, 2019

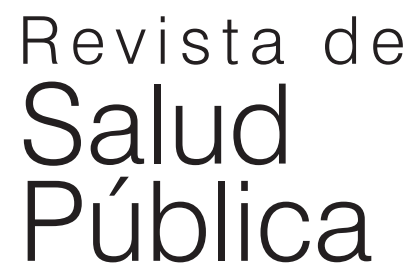




\section{Sumario}

EDITORIAL

PALABRAS DE LA VISE DECANA

\section{ARTICULOS ORIGINALES}

Salud adolescente y la mirada de los actores institucionales en cinco Provincias del norte Argentino. Paradigmas, percepciones y programas.

Daniel Maceira, Cintia Hasicic, Milva Carlino, Marcelo Ibarra, Paola Marcos, Damián Navarro, Fernando Sadir.

Coordinación de la atención entre niveles y sus factores asociados en dos subredes de la red municipal de salud de la Ciudad de Rosario, Argentina.

Julia Puzzolo, Delia Inés Amarilla, Marisel Colautti, Justina Moreno, Pierre De Paepe, Lorenzo Vargas, Ingrid Vázquez, María Luisa Navarrete.

Queratitis por acanthamoeba. Infección ocular emergente. Reporte de casos.

Roque A. Maffrand, Rogelio Daniel Pizzi, Mirtha G. Páez R., Axel Tomas, Carola M. Maffrand, Hugo Luis Pizzi.

Una mirada a los sistemas de salud Mexicano y Argentino.

Rosa Amarilis Zárate Grajales, Zoila Romualdo Pérez, Lucrecia Guadalupe Sánchez Bañuelos, Noemí García Hernández, Anayeli Villegas Zúñiga, Diana Lizbeth Alvarado Celaya.

Salud para todos: conceptualización y perspectivas.

Noemí Bordoni, Aldo Squassi.

\section{FORO ABIERTO DE OPINION}

Aspectos psicológicos en niños con labio leporino y/o paladar hendido: un breve comentario sobre algunos hallazgos relevantes para los equipos de salud.

Marcos Roberto Tovani Palone 


\section{Summary}

EDITORIAL

\section{PALABRAS DE LA VISE DECANA}

\section{ARTICULOS ORIGINALES}

Adolescent health and the look of institutional actors in five Provinces in the north of Argentina. Paradigms, perceptions and programs.

Daniel Maceira, Cintia Hasicic, Milva Carlino, Marcelo Ibarra, Paola Marcos, Damián Navarro, Fernando Sadir.

Coordination of care between levels and their associated factors in two subnets of the municipal health network in the City of Rosario, Argentina.

Julia Puzzolo, Delia Inés Amarilla, Marisel Colautti, Justina Moreno, Pierre De Paepe, Lorenzo Vargas, Ingrid Vázquez, María Luisa Navarrete.

Acanthamoeba keratitis. Emerging ocular infection. Case reports.

Roque A. Maffrand, Rogelio Daniel Pizzi, Mirtha G. Páez R., Axel Tomas, Carola M. Maffrand, Hugo Luis Pizzi.

A look to Mexican and Argentinian health systems.

Rosa Amarilis Zárate Grajales, Zoila Romualdo Pérez, Lucrecia Guadalupe Sánchez Bañuelos, Noemí García Hernández, Anayeli Villegas Zúñiga, Diana Lizbeth Alvarado Celaya.

Health for everybody. Conceptualization and perspectives.

Noemí Bordoni, Aldo Squassi.

\section{OPEN OPINION FORUM}

Psychological aspects in children with cleft lip and/or palate: a brief commentary onsome relevant findings for health teams.

Marcos Roberto Tovani Palone 


\section{ARTICULOS ORIGINALES}

Saúde adolescente e o olhar dos atores institucionais em cinco províncias do norte Argentino. Paradigmas, percepções e programas.

Daniel Maceira, Cintia Hasicic, Milva Carlino, Marcelo Ibarra, Paola Marcos, Damián Navarro, Fernando Sadir.

Coordenação de cuidados entre os níveis e fatores associados em dois sub-redes da rede municipal de saúde da Cidade de Rosario, Argentina.

Julia Puzzolo, Delia Inés Amarilla, Marisel Colautti, Justina Moreno, Pierre De Paepe, Lorenzo Vargas, Ingrid Vázquez, María Luisa Navarrete.

Ceratite por acanthamoeba. Infecção ocular emergente. Relatório dos caso.

Roque A. Maffrand, Rogelio Daniel Pizzi, Mirtha G. Páez R., Axel Tomas, Carola M. Maffrand, Hugo Luis Pizzi.

Um olhar para os sistemas de saúde Mexicanos e Argentinos.

Rosa Amarilis Zárate Grajales, Zoila Romualdo Pérez, Lucrecia Guadalupe Sánchez Bañuelos, Noemí García Hernández, Anayeli Villegas Zúñiga, Diana Lizbeth Alvarado Celaya.

Saúde para todos: conceptualização e perspectivas.

Noemí Bordoni, Aldo Squassi.

FÓRUM DE OPINIÃO ABERTA

Aspetos psicológicos em crianças com lábio leporino e / ou fenda palatina: um comentário breve sobre algumas conclusões marcantes para as equipes de saúde. Marcos Roberto Tovani Palone 


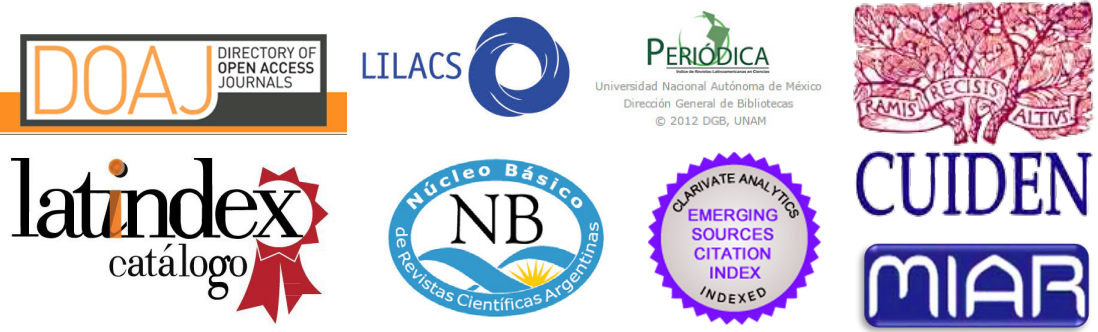

BIBLIOTECA ELECTRÓNICA DE CIENCIA Y TECNOLOGÍA
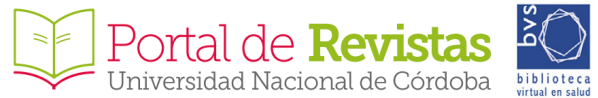

\section{Revista indizada en base de datos}

LILACS

Literatura Latinoamericana y del Caribe en Ciencias de la Salud. Bireme, Sistema Latinoamericano y del Caribe de Información en Ciencias de la Salud, Brasil. http://www.bireme.br/

\section{CUIDEN}

Base de Datos Bibliográfica sobre Cuidados de Salud en Iberoamérica. Base de datos de enfermería en español, Granada - España http://www.doc6.es/index/

\section{PERIODICA}

Base de datos de la Dirección General de Bibliotecas de la UNAM. Universidad Nacional Autónoma de México. http://132.248.9.1:8991/F/-/?func=find-b0\&local base $=$ PER 01

\section{RENICS}

Red Nacional de Información en Ciencias de la Salud. http://www.renics.com.ar/

DOAJ

Directory of open access journals Universidad Lund, Suecia http://www.doaj.org/

NÚCLEO BÁSICO DE REVISTAS CIENTÍFICAS ARGENTINAS. CAYCITCONICET. R. Argentina HTTP://www.caicyt-conicet.gov.ar/nucleo-basico-derevistas-cientificas/

SISTEMA NACIONAL DE REPOSITORIOS DIGITALES - Biblioteca Electrónica de Ciencia y Tecnología del Ministerio de Ciencia, Tecnología e Innovación Productiva (Mincyt). Se accede a través de este link:

http://www.biblioteca.mincyt.gob.ar/revistas/index?letter $=\&$ title $=$ revista $+\mathrm{de}+\mathrm{s}$ alud + publica\&openaccess $=$ false\&gran_area $=3 \&$ area $=\& v i e w=l i s t \& y t 0=$ Busca

Revistas de la Universidad Nacional de Córdoba espacio destinado a revistas electrónicas, de acceso librema la produccion cientifica, un recurso publico, es por ello que somos parte de este sitio que la universidad ofrece de acceso libre a la produc

El sistema con que opera este sitio es Open Journal Systems (OJS).

Se accede: http://revistas.unc.edu.ar/ 